Pacific

Journal of

Mathematics

THE PRODUCT OF EXPONENTIALS IN THE DEFINITION OF CANONICAL KERNEL FUNCTION

M.P. DE Oliveira And J.A. Verderesi 


\title{
THE PRODUCT OF EXPONENTIALS IN THE DEFINITION OF CANONICAL KERNEL FUNCTION
}

\author{
M.P. De Oliveira AND J.A. Verderesi
}

\begin{abstract}
We calculate the Campbell-Baker-Hausdorff series for the product of two exponentials appearing in the usual definition of canonical kernel function.
\end{abstract}

\section{Introduction.}

The canonical kernel function of a bounded symmetric domain $\mathcal{D}$ in its Harish-Chandra realization has been introduced by I. Satake in $[\mathbf{8}, \mathbf{9}, \mathbf{1 0}, \mathbf{1 1}]$ and since then it has found several applications. Some of them would include reproducing kernels for Hilbert spaces of holomorphic functions on $\mathcal{D}$ associated with the holomorphic discrete series representations, the commutator of certain elements in the universal enveloping algebra of a complex semisimple Lie algebra of hermitian type, expressions for the contravariant form on a highest weight module over such a Lie algebra, among others (see $[\mathbf{1}, \mathbf{2}])$.

We will not comment on the importance of the Campbell-Baker-Hausdorff series. Even though Goldberg's formulas provide an algorithm to figure out the coefficients of the series by recurrence, only in very few particular cases they are "explicitly" known in terms of "sufficiently elementary functions". Moreover, as in the present case, there are certain linear relations among the Lie monomials which are exclusive to the Lie algebra under consideration and not regarded in the general setting.

From this point of view, it seems reasonable to us to work out the series for the product of two exponentials like those in the definition of canonical kernel function.

\section{Preliminaries.}

Let $\mathfrak{g}$ be a real semisimple Lie algebra of hermitian type. By hermitian type we mean that for some, and thus for any, Cartan decomposition $\mathfrak{g}=\mathfrak{k}+\mathfrak{p}$ there is an element $H_{0}$ in the center $\mathfrak{c}$ of $\mathfrak{k}$ such that ad $H_{0}$ is a complex structure on $\mathfrak{p}$. Fix one of them and let $\theta$ be the corresponding Cartan involution.

Let $\mathfrak{c}$ be the center of $\mathfrak{k}$ and choose $\mathfrak{h} \subset \mathfrak{g}$ a Cartan subalgebra of $\mathfrak{g}$ contained in $\mathfrak{k}$. Let $\mathfrak{g}_{c}, \mathfrak{k}_{c}, \mathfrak{c}_{c}, \mathfrak{h}_{c}, \mathfrak{p}_{c}$ be the complexifications of $\mathfrak{g}, \mathfrak{k}, \mathfrak{c}, \mathfrak{h}, \mathfrak{p}$ and 
$\mathfrak{p}_{+}, \mathfrak{p}_{-}$the eigenspaces of $\operatorname{ad}_{\mathfrak{p}_{c}} H_{0}$ with eigenvalues $i$ and $-i$, respectively. Denote with $\mathfrak{B}$ the Killing form of $\mathfrak{g}_{c}$.

Let $G_{c}$ be the connected simply connected Lie group with Lie algebra $\mathfrak{g}_{c}$ and $P_{+}, P_{-}, K_{c}, G, K$ the analytic subgroups of $G_{c}$ with Lie algebras $\mathfrak{p}_{+}, \mathfrak{p}_{-}$, $\mathfrak{k}_{c}, \mathfrak{g}$ and $\mathfrak{k}$ respectively. The exponential map of $G_{c}$ induces a holomorphic diffeomorphism of $\mathfrak{p}_{+}\left(\mathfrak{p}_{-}\right)$onto $P_{+}\left(P_{-}\right)$(see $[\mathbf{6}]$ ).

By a theorem of Harish-Chandra, the map

$$
\psi: P_{+} \times K_{c} \times P_{-} \rightarrow G_{c}, \psi(p, k, q)=p k q
$$

is a holomorphic diffeomorphism onto an open dense subset of $G_{c}$ and $G \subset P_{+} K_{c} P_{-}$. In general, given $g \in \operatorname{Im} \psi$, we write $g=g_{+} g_{0} g_{-}$for the " $P_{+} K_{c} P_{-}$" decomposition of $g$, where $g_{+} \in P_{+}, g_{0} \in K_{c}$ and $g_{-} \in P_{-}$. By analogy, these results can be reformulated for a " $P_{-} K_{c} P_{+}$" decomposition.

Now, it follows from the results mentioned above that we have the open holomorphic embeddings:

$$
\mathcal{D}=G / K \simeq G K_{c} P_{-} / K_{c} P_{-} \hookrightarrow P_{+} K_{c} P_{-} / K_{c} P_{-} \simeq P_{+} \simeq \mathfrak{p}_{+} .
$$

One can prove that the image of the composition of these embeddings is a bounded and holomorphically symmetric connected open subset of $\mathfrak{p}_{+}$which is known as the Harish-Chandra realization of $\mathcal{D}$ as a bounded symmetric domain (see [11]).

\section{The canonical kernel function.}

We define the canonical kernel function of a bounded symmetric domain in its Harish-Chandra realization as

$$
\kappa: \mathcal{D} \times \mathcal{D} \rightarrow K_{c}, \quad \kappa(z, w)=\left((\exp -\bar{w} \exp z)_{0}\right)^{-1} \in K_{c},
$$

which is well-defined on $\mathcal{D} \times \mathcal{D}$ because, for $z, w \in \mathcal{D}$,

$$
\exp -\bar{w} \exp z \in \overline{G K_{c} P_{-}} G K_{c} P_{-}=P_{+} K_{c} G K_{c} P_{-}=P_{+} K_{c} P_{-} .
$$

In other words, $\kappa(z, w)$ is the inverse of the $K_{c}$-part of

$$
\exp -\bar{w} \exp z
$$

in the " $P_{+} K_{c} P_{-}$" decomposition or, alternatively, the $K_{c}$-part of

$$
\exp -z \exp \bar{w}
$$

in the " $P_{-} K_{c} P_{+}$" decomposition.

In what follows, we are concerned about rewriting either of the products (3.2) or (3.3) as a single exponential. Notice that they represent the same problem since an expression for one of them can readily be obtained from an expression for the other (in this case by means of the inverse operation). 


\section{Main results.}

Lemma 4.1. Let

$$
E=\left(\begin{array}{ll}
0 & 1 \\
0 & 0
\end{array}\right), \quad F=\left(\begin{array}{cc}
0 & 0 \\
1 & 0
\end{array}\right)
$$

Then

$$
\exp t E \exp t F=\exp \mathcal{C}\left(t^{2}\right)\left(t E+\frac{1}{2}[t E, t F]+t F\right), t \in \mathbb{R}
$$

where $\mathcal{C}:(-4, \infty) \longrightarrow \mathbb{R}$ is the function given by

$$
\begin{gathered}
\mathcal{C}(u)=\sum_{n \geqslant 0} \frac{(-1)^{n}}{n+1}\left(\begin{array}{c}
2 n+1 \\
n
\end{array}\right)^{-1} u^{n}, \quad|u|<4, \\
\mathcal{C}(u)=2 \frac{\log \left(1+u / 2+\sqrt{u^{2}+4 u} / 2\right)}{\sqrt{u^{2}+4 u}}, \quad u>0 .
\end{gathered}
$$

Proof. Let

$$
A=\left(\begin{array}{cc}
t^{2} & t \\
t & 0
\end{array}\right)
$$

(4.4) $\log (\exp t E \exp t F)=\log \left(\left(\begin{array}{ll}1 & t \\ 0 & 1\end{array}\right)\left(\begin{array}{ll}1 & 0 \\ t & 1\end{array}\right)\right)=\log (I+A)$.

For each $t \in \mathbb{R}$, the matrix $A$ is symmetric with eigenvalues

$$
\lambda_{+}=\frac{1}{2}\left(t^{2}+t \sqrt{t^{2}+4}\right) \quad \lambda_{-}=\frac{1}{2}\left(t^{2}-t \sqrt{t^{2}+4}\right)
$$

and, if $t \neq 0, A$ has corresponding orthonormal eigenvectors

$$
\begin{aligned}
& v_{+}=\left(\lambda_{+} / \sqrt{t^{2}+\lambda_{+}^{2}}, t / \sqrt{t^{2}+\lambda_{+}^{2}}\right) \\
& v_{-}=\left(\lambda_{-} / \sqrt{t^{2}+\lambda_{-}^{2}}, t / \sqrt{t^{2}+\lambda_{-}^{2}}\right)
\end{aligned}
$$

forming the columns of the matrix

$$
M=\left(\begin{array}{ll}
\lambda_{+} / \sqrt{t^{2}+\lambda_{+}^{2}} & \lambda_{-} / \sqrt{t^{2}+\lambda_{-}^{2}} \\
t / \sqrt{t^{2}+\lambda_{+}^{2}} & t / \sqrt{t^{2}+\lambda_{-}^{2}}
\end{array}\right) .
$$

Let $D(a, b)$ be the diagonal matrix whose diagonal is $(a, b)$, from the top. Then for sufficiently small values of $t$

$$
\begin{aligned}
\log (I+A) & =\log \left(I+M D\left(\lambda_{+}, \lambda_{-}\right) M^{T}\right) \\
& =M D\left(\log \left(1+\lambda_{+}\right), \log \left(1+\lambda_{-}\right)\right) M^{T}
\end{aligned}
$$

proving formula (4.1), with $\mathcal{C}$ given by (4.3), locally around zero, $t \neq 0$. 
To prove the development into series (4.2), maybe it is easier now to develop one of the entries of expression (4.4) directly. It is worth listing the entry at position $(1,2)$ for some of the powers of $A$ :

$$
\begin{aligned}
& A(1,2)=t \\
& A^{2}(1,2)=t^{3} \\
& A^{3}(1,2)=t^{5}+t^{3} \\
& A^{4}(1,2)=t^{7}+2 t^{5} \\
& A^{5}(1,2)=t^{9}+3 t^{7}+t^{5} \\
& A^{6}(1,2)=t^{11}+4 t^{9}+3 t^{7} \\
& A^{7}(1,2)=t^{13}+5 t^{11}+6 t^{9}+t^{7} \\
& \begin{array}{rll}
A^{8}(1,2) & =t^{15}+6 t^{13}+10 t^{11}+4 t^{9} \\
\vdots & = & \ddots
\end{array}
\end{aligned}
$$

Figure 1. $(1,2)$-entry for powers of $A$.

One has

$$
A^{2}=t^{2}(A+I) \text { and hence } A^{m}=t^{2}\left(A^{m-1}+A^{m-2}\right), m \geqslant 2 .
$$

From above expression, it follows

$$
\begin{aligned}
A^{m}(1,2)= & \sum_{n=0}^{\infty} C_{m}^{2 n+1} t^{2 n+1}, m \geqslant 1, \text { where } C_{m}^{2 n+1}=0 \text { for } n \geqslant m . \\
& C_{m}^{2 n+1}=C_{m-1}^{2 n-1}+C_{m-2}^{2 n-1}, m \geqslant 3, n \geqslant 1 .
\end{aligned}
$$

Now, define $B_{p}^{n}$ as

$$
B_{p}^{n} \equiv C_{p+n+1}^{2 n+1}, n \geqslant 0, p \geqslant 0 .
$$

Since

$$
\begin{gathered}
B_{p}^{n}=B_{p}^{n-1}+B_{p-1}^{n-1}, n \geqslant 1, p \geqslant 1, \\
B_{p}^{0}=C_{p+1}^{1}=0, p \geqslant 1, B_{0}^{0}=1, \\
B_{0}^{n}=C_{n+1}^{2 n+1}=1, n \geqslant 0,
\end{gathered}
$$

one has

$$
B_{p}^{n}=\left(\begin{array}{c}
n \\
p
\end{array}\right), n \geqslant 0, p \geqslant 0 .
$$

Because of the identity (see [4])

$$
\sum_{m=0}^{n}\left(\begin{array}{c}
n \\
m
\end{array}\right) \frac{(-1)^{m}}{x+m}=x^{-1}\left(\begin{array}{c}
x+n \\
n
\end{array}\right)^{-1}, \quad x \notin\{0,-1, \cdots-n\},
$$


it follows for small enough values of $t$

$$
\log (I+A)(1,2)=\sum_{n \geqslant 0} \frac{(-1)^{n}}{n+1}\left(\begin{array}{c}
2 n+1 \\
n
\end{array}\right)^{-1} t^{2 n+1},
$$

and therefore $\mathcal{C}$ can be given by series (4.2), locally above zero. By analytic continuation, $\mathcal{C}$ can be extended to $(-4, \infty)$ as described in the statement. Also by analyticity, expression (4.1) is valid for any $t \in \mathbb{R}$.

Remark 4.2. The last expression in Lemma 4.1 (Formula (4.3)) can alternatively be obtained noticing that

$$
B=\log (I+A)=\left(\begin{array}{rr}
x & y \\
y & -x
\end{array}\right)
$$

satisfies

$$
\exp B=I+A=\cosh (r) I+\frac{\sinh (r)}{r} B
$$

where $r=\sqrt{x^{2}+y^{2}}$.

Theorem 4.3 (Campbell-Baker-Hausdorff formula for $z \in \mathfrak{p}_{+}$and $w \in \mathfrak{p}_{-}$).

$$
\exp z \exp w=\exp \mathcal{H}(z, w) \text {, where }
$$

$\mathcal{H}(z, w)=\mathcal{C}\left(\frac{1}{2} \operatorname{ad} z \operatorname{ad} w\right) z+\frac{1}{2} \mathcal{C}\left(\frac{1}{2} \operatorname{ad} z \operatorname{ad} w\right)[z, w]+\mathcal{C}\left(\frac{1}{2} \operatorname{ad} w \operatorname{ad} z\right) w$ for $(z, w) \in \mathfrak{p}_{+} \times \mathfrak{p}_{-}$such that $\| \operatorname{ad} z$ ad $w / 2 \|<4$. Here the norm \|\| is the operator norm associated with the positive definite hermitian form $-\mathfrak{B}(X, \theta \bar{Y})$ on $\mathfrak{g}_{\mathrm{c}}$ and $C(X)$ denotes the series

$$
\mathcal{C}(X)=\sum_{n \geqslant 0} \frac{(-1)^{n}}{n+1}\left(\begin{array}{c}
2 n+1 \\
n
\end{array}\right)^{-1} X^{n}
$$

where $X: \mathfrak{g}_{c} \rightarrow \mathfrak{g}_{c}$ is a linear operator.

Proof. It is a corollary of Lemma 4.1. We use the strongly orthogonal root technique: Fix a basis $\Phi$ for the root system $\Delta$ of $\mathfrak{g}_{c}$ relative to $\mathfrak{h}_{c}$ such that

$$
\mathfrak{p}_{+}=\sum_{\alpha \in \Delta_{n}^{+}} \mathfrak{g}_{\alpha} \quad \mathfrak{p}_{-}=\sum_{\alpha \in \Delta_{n}^{+}} \mathfrak{g}_{-\alpha}
$$

where $\Delta_{n}^{+}$is the set of positive noncompact roots. Now we can also denote with $\Delta^{+}, \Delta_{c}^{+}$the sets of positive roots and positive compact roots respectively.

First, suppose that $\mathfrak{g}_{c}$ is a simple Lie algebra. Then there is exactly one positive noncompact simple root. Recall that two distinct roots are strongly 
orthogonal if neither their sum nor difference is a root. Form the maximal sequence

$$
\Gamma=\gamma_{1}, \ldots, \gamma_{s}
$$

such that $\gamma_{1}$ is the unique noncompact simple root and $\gamma_{j}$ is the lowest positive noncompact root strongly orthogonal to $\gamma_{1}, \ldots, \gamma_{j-1}, j=2, \ldots, s$ (there exists such a lowest element in the partial order prescribed by $\Phi$ ). In case $\mathfrak{g}_{c}=\bigoplus_{i=1}^{m} \mathfrak{g}_{i}$ is a sum of simple ideals, consider $\Gamma_{i}$ as the sequence of strongly orthogonal roots of $\mathfrak{g}_{i}$ constructed as above and define

$$
\Gamma=\gamma_{1}, \ldots, \gamma_{s}
$$

as the sequence obtained concatenating $\Gamma_{1}, \Gamma_{2}, \ldots, \Gamma_{m}$.

By a result of Harish-Chandra, the number of roots in this sequence equals the dimension of any maximal abelian subalgebra of $\mathfrak{p}$.

Choose elements $E_{\beta} \in g_{\beta}, E_{-\beta} \in g_{-\beta}$, such that $\bar{E}_{\beta}=E_{-\beta}$ and normalize them in such a way $\left[\left[E_{\beta}, E_{-\beta}\right], E_{\beta}\right]=2 E_{\beta}, \beta \in \Delta_{n}^{+}$. From the first condition it follows that $\sum_{i=1}^{s} \mathbb{R}\left(E_{\gamma_{i}}+E_{-\gamma_{i}}\right)$ is a maximal abelian subalgebra of $\mathfrak{p}$. One has from the second one that $E_{\beta}, E_{-\beta}$ and $H_{\beta}=\left[E_{\beta}, E_{-\beta}\right]$ form a Lie algebra isomorphic to $\mathfrak{s l}(2, \mathbb{C})$, for $\pm \beta \in \Delta_{n}^{+}$. From Lemma 4.1 and the fact that the group $S L(2, \mathbb{C})$ is simply connected, it follows

$$
\exp t_{i} E_{\gamma_{i}} \exp t_{i} E_{-\gamma_{i}}=\exp \mathcal{C}\left(t_{i}^{2}\right)\left(t_{i} E_{\gamma_{i}}+\frac{1}{2}\left[t_{i} E_{\gamma_{i}}, t_{i} E_{-\gamma_{i}}\right]+t_{i} E_{-\gamma_{i}}\right)
$$

where $t_{i}$ are real numbers, $i=1, \ldots, s$. Now for

$$
z=\sum_{i=1}^{s} t_{i} E_{\gamma_{i}}
$$

one has

$$
\bar{z}=\sum_{i=1}^{s} t_{i} E_{-\gamma_{i}} .
$$

By strongly orthogonality of the roots $\gamma_{i}$, we conclude that for small $\left|t_{i}\right|$

$$
\exp z \exp \bar{z}=\exp \mathcal{H}(z, \bar{z}), \quad \text { where }
$$

$\mathcal{H}(z, \bar{z})=\mathcal{C}\left(\frac{1}{2} \operatorname{ad} z \operatorname{ad} \bar{z}\right) z+\frac{1}{2} \mathcal{C}\left(\frac{1}{2} \operatorname{ad} z \operatorname{ad} \bar{z}\right)[z, \bar{z}]+\mathcal{C}\left(\frac{1}{2} \operatorname{ad} \bar{z} \operatorname{ad} z\right) \bar{z}$.

Since for any $z \in \mathfrak{p}_{+}$, there is $k \in K$ such that

$$
z=\operatorname{Ad} k\left(\sum_{i=1}^{s} t_{i} E_{\gamma_{i}}\right),
$$

expression (4.17) is actually valid for $z \in \mathfrak{p}_{+}$, locally at zero. 
Because of this, the equality to be proved is valid locally at zero, since both of its sides are holomorphic in $z$ and antiholomorphic in the auxiliary variable $z_{2} \in \mathfrak{p}_{+}$such that $w=\bar{z}_{2}$. But then they coincide on the (connected) subset

$$
\Omega=\left\{(z, w) \in \mathfrak{p}_{+} \times \mathfrak{p}_{-} \mid\left\|\frac{1}{2} \operatorname{ad} z \operatorname{ad} w\right\|<4\right\} .
$$

Remark 4.4. In above notation, one can describe the bounded symmetric domain $\mathcal{D}$ and the canonical kernel function in "coordinates" as

$$
\begin{gathered}
\mathcal{D}=\left\{\operatorname{Ad} k\left(\sum_{i=1}^{s} t_{i} E_{\gamma_{i}}\right)\left|k \in K, t_{i} \in \mathbb{R},\right| t_{i} \mid<1\right\} \quad(\text { see }[5]), \\
\kappa\left(\sum_{i=1}^{s} t_{i} E_{\gamma_{i}}, \sum_{i=1}^{s} u_{i} E_{\gamma_{i}}\right)=\exp \sum_{i=1}^{s} \log \left(1-t_{i} u_{i}\right)\left[E_{\gamma_{i}}, E_{-\gamma_{i}}\right], \\
t_{i}, u_{i} \in \mathbb{R},\left|t_{i}\right|,\left|u_{i}\right|<1 \quad(\mathrm{cf}[\mathbf{1 2}]) .
\end{gathered}
$$

They confirm that, in fact, the canonical kernel function is defined on the subset $\mathcal{D} \times \mathcal{D}$ of $\mathfrak{p}_{+} \times \mathfrak{p}_{+}$but cannot be extended to all points of its closure.

On the other hand, the bounded symmetric domain can alternatively be characterized as

$$
\mathcal{D}=\left\{z \in \mathfrak{p}_{+} \mid\|\operatorname{ad} z\|<\sqrt{2}\right\} \quad(\text { see }[\mathbf{1}]),
$$

which means that the domain $\Omega$ contains pairs of points where the canonical kernel function's expression is not defined, for instance, for pairs $(z, \bar{z})$ such that $z$ lies on the boundary of $D$.

Remark 4.5. Equality (4.14) is still valid if we consider $\mathfrak{g}(z, w)$, the free 3 -graded Lie algebra generated by variables $z$ of degree 1 and $w$ of degree -1 . In this case, series (4.15) and the exponentials above are formal series with terms in $\mathfrak{g}(z, w)$ and $\mathfrak{U}(\mathfrak{g}(z, w))$, the universal enveloping algebra of $\mathfrak{g}(z, w)$, respectively (see $[\mathbf{2}])$.

Remark 4.6. The results above can also be obtained via Goldberg's recurrently computable coefficients of the commutator-free $\log \left(e^{x} e^{y}\right)$, as in [3], but one still has to take into account the dependence among the terms which are specific to $\mathfrak{g}_{c}$. To obtain series (4.15), for instance, it is enough to consider the identities:

i) $(\operatorname{ad} z)^{3}=(\operatorname{ad} w)^{3}=0$,

ii) $\operatorname{ad} z \operatorname{ad} w(\operatorname{ad} z \operatorname{ad} w)^{k}[z, w]=\operatorname{ad} w \operatorname{ad} z(\operatorname{ad} z \operatorname{ad} w)^{k}[z, w], \quad k \geqslant 0$, for $z \in \mathfrak{p}_{+}$and $w \in \mathfrak{p}_{-}$. The relations in the second family can be proved using the strongly orthogonal root technique, as in the proof of (4.14). 


\section{References}

[1] M. De Oliveira, Some formulas for the canonical kernel function, Geom. Dedicata, 86(1) (2001), 227-247, CMP 1856428.

[2] _ On commutation relations for 3-graded Lie algebras, New York J. Math., 7 (2001), 71-86, CMP 1856712.

[3] K. Goldberg, The formal power series for $\log \left(e^{x} e^{y}\right)$, Duke Math. J., 23 (1956), 13-21, MR 18,572f, Zbl 0070.25203.

[4] R. Graham, D. Knuth and O. Patashnik, Concrete Mathematics: A Foundation for Computer Science, second ed., Reading, Addison Wesley, 1994, MR 97d:68003, Zbl 0836.00001.

[5] Harish-Chandra, Representations of semisimple Lie groups VI. Integrable and squareintegrable representations, Amer. J. Math., 78 (1956), 564-628, MR 18,490d, Zbl 0072.01702.

[6] S. Helgason, Differential Geometry, Lie Groups and Symmetric Spaces, Academic Press, New York, 1978, MR 80k:53081, Zbl 0451.53038.

[7] A. Korányi and J.A. Wolf, Realization of hermitian symmetric spaces as generalized half-planes, Ann. of Math., 81 (1965), 265-288, MR 30 \#4980, Zbl 0137.27402.

[8] I. Satake, On unitary representations of a certain group extension, Sugaku, Math. Soc., Japan, 21 (1969), 241-253, MR 43 \#2164.

[9] , Factors of automorphy and Fock representations, Adv. in Math., 7(2) (1971), 83-110, MR 50 \#541, Zbl 0219.22016.

[10] _ Fock representations and theta functions, in 'Advances in the Theory of Riemann Surfaces,' Ann. Math. Studies, 66, Princeton Univ. Press, 1971, 393-405, MR 54 \#12657, Zbl 0217.43301.

[11] Algebraic Structures of Symmetric Domains, Iwanami Shoten, Tokyo and Princeton University Press, Princeton, NJ, 1980, MR 82i:32003, Zbl 0483.32017.

[12] N. Wallach, The analytic continuation of the discrete series I, Trans. Amer. Math. Soc., 251 (1979), 1-17, MR 81a:22009, Zbl 0419.22017.

Received November 1, 2000 and revised January 14, 2002. Research partially supported by FAPESP.

Department of Mathematics

UNIVERSITY OF TORONTO

TORONTO, Ontario M5S 3G3

CANADA

E-mail address: mpdeoliv@math.toronto.edu

Department of Mathematics

UNIVERSity OF SÃo PAUlo

BRAzIL

E-mail address: javerd@ime.usp.br 